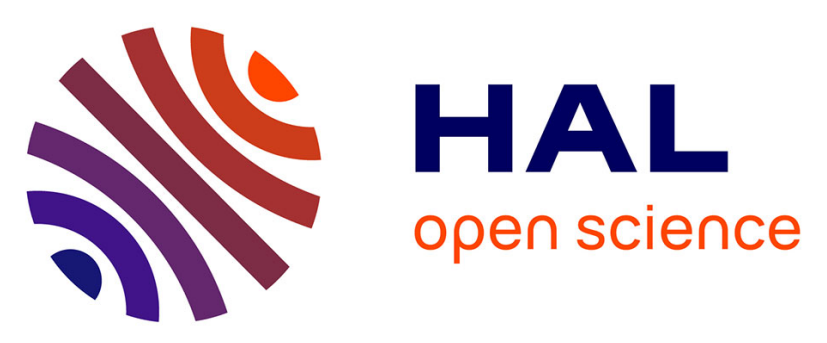

\title{
Static and dynamic analysis of a 6 DoF totally constrained cable robot with 8 preloaded cables
}

Damien Gueners, B. Chedli Bouzgarrou, Hélène Chanal

\section{To cite this version:}

Damien Gueners, B. Chedli Bouzgarrou, Hélène Chanal. Static and dynamic analysis of a 6 DoF totally constrained cable robot with 8 preloaded cables. Andreas Pott; Tobias Bruckmann. CableDriven Parallel Robots. Proceedings of the 4th International Conference on Cable-Driven Parallel Robots, 74, Springer International Publishing, pp.307-318, 2019, Mechanisms and Machine Science, 978-3-030-20750-2. 10.1007/978-3-030-20751-9_26. hal-03384959

\section{HAL Id: hal-03384959 \\ https://hal.science/hal-03384959}

Submitted on 19 Oct 2021

HAL is a multi-disciplinary open access archive for the deposit and dissemination of scientific research documents, whether they are published or not. The documents may come from teaching and research institutions in France or abroad, or from public or private research centers.
L'archive ouverte pluridisciplinaire HAL, est destinée au dépôt et à la diffusion de documents scientifiques de niveau recherche, publiés ou non, émanant des établissements d'enseignement et de recherche français ou étrangers, des laboratoires publics ou privés. 


\title{
Static and dynamic analysis of a 6 DoF totally constrained cable robot with 8 preloaded cables
}

\author{
Damien Gueners ${ }^{1}$, B. Chedli Bouzgarrou ${ }^{1}$ and Hélène Chanal ${ }^{1}$ \\ ${ }^{1}$ Université Clermont Auvergne, CNRS, SIGMA Clermont, Institut Pascal, F-63000 Clermont- \\ Ferrand, France \\ damien.guenersesigma-clermont.com
}

\begin{abstract}
In this paper, the static and dynamic performances of a cable-driven parallel robot (CDPR) are analyzed over all its entire workspace. The considered robot has 6 degrees of freedom and it is completely constrained with 8 cables. This paper aims at highlighting the effects of cable preloads on the robot's behavior. To this end, the stiffness matrix of the robot is computed from a second order approximation of the deformation energy of preloaded cables. Lagrange formulation is used to obtain dynamic equations of the robot for vibration mode calculation and trajectory tracking simulations. Static and modal analyses show that, for a given preload, the increase in the robot's stiffness and modal frequencies is relatively more significant using soft cables, made of nylon, rather than stiff cables made of steel. These results are confirmed by dynamic simulations illustrating the effects of preloaded cables on the accuracy of trajectory tracking performance.
\end{abstract}

Keywords: Stiffness, Vibration, Cable preload.

\section{Introduction}

CDPRs [1] are robots for which the fixed base and the mobile platform are connected by cables. There are no guiding elements except those of the pulleys used to wind and unwrap the cables. The main advantages of this technology are:

- reduction of the moving masses resulting in a large acceleration capacity of the moving platform;

- reconfigurability and modularity: the robot can be reconfigured easily by changing the anchor points of the actuators and adapting its geometric control model;

- reduction of the production costs.

The static and dynamic rigidity of cable robots is generally lower than that of conventional parallel robots due to the flexibility of the cables. The accuracy and vibration stability of existing cable robots is not high enough to consider them for dynamic loading applications such as the machining process.

CDPRs can be divided into 3 main families:

- Suspended cable driven parallel robots[2] where all the points of attachment are above the mobile platform. Gravity plays the role of a force cable that pulls down.

- Completely constrained cable driven parallel robots[3] that have cables above and below the mobile platform. 
- Hybrid parallel cable robots[4] that have cables which guide a rigid arm.

Some research has been done on the stiffness and vibration analysis of cable robots. In [5], it has been shown that the transverse vibrations of cables can be negligible in relation to the total vibrations of the effector in comparison to their axial vibrations. For a fully constrained robot, $98.6 \%$ of the mobile platform vibrations are caused by axial vibrations of the cables. These results have also been used to approve the modeling of the cables by axial springs for totally constrained CDPRs. In [6], the longitudinal and lateral vibration on a planar and a cable suspended robot is studied by using a finite element method. In [7, 8], the dynamic stiffness matrix is proposed to analyze the vibration of a cable suspended robot with a sagging cable model. In [9], the control of reaction wheels mounted on the mobile platform has been used to compensate its vibrations. In [10], a controller has been proposed to attenuate the transverse vibrations of a planar CDPR, which has a lower stiffness in the axis perpendicular to the plane motion.

The paper cited above did not however study precisely the influence of cable preload on the performance of cable robots. In this paper we consider a totally constrained cable robot, with $6 \mathrm{DOFs}$, driven by 8 preloaded cables. The proposed research is carried out within the CABFAB project, which addresses the design of a CDPR for additive manufacturing processes. The presented work is part of a preliminary design phase, aimed at guaranteeing a minimal level of rigidity over the entire robot workspace, to be optimized in terms of accessibility in position and orientation. For additive manufacturing, a high level of accuracy for tool path tracking is required. A completely constrained cable robot should have reach a higher level of accuracy than a suspended one [1]. Moreover, since the stiffness depends directly on the cable lengths, a medium-scale robot of $1 \mathrm{~m}^{3}$ size, as that considered in this paper, can have a relatively higher accuracy compared to the large-scale cable robots.

This paper investigates the influence of cable preloads on the stiffness and vibration modes of a CDPR. First, the kinematic and dynamic models are presented. The static stiffness and modal frequencies are then determined for various cable preloads. Next the static and dynamic simulation of a completely constrained CPDR are analyzed. The stiffness and natural mode inside the feasible workspace are studied. The static stiffness and natural frequency are compared for different preloads and cables. The robot's dynamic accuracy is evaluated as a function of the cable preload.

\section{Robot modeling}

For the considered application, the robot can incorporate thin cables with the aim of printing medium-sized parts at high speeds. Therefore, the mass of the cable can be considered as negligible and the effect of sagging is less significant than with large CDPRs[2]. Thus, the kinematic model does not take into account the sagging effect. In addition, cable deformations rely on linear elasticity assumptions. The inverse kinematic model will be presented in this section. 


\subsection{Inverse Kinematics}

The geometric model uses $m$ cables of length $l_{i}$ with one anchor point on the mounting frame in $A_{i}$, and another anchor point on the end-effector in $B_{i}$ (fig.1). $\mathcal{R}_{O}\left(O, \mathbf{e}_{x_{O}}, \mathbf{e}_{y_{O}}, \mathbf{e}_{z_{O}}\right)$ is the fixed frame of reference and $\mathcal{R}_{E}\left(C, \mathbf{e}_{x_{E}}, \mathbf{e}_{y_{E}}, \mathbf{e}_{z_{E}}\right)$ is the frame attached to the mobile platform at its characteristic point $C$. The kinematic constraints of the system can be given by the vector loop-closure equation for each cable:

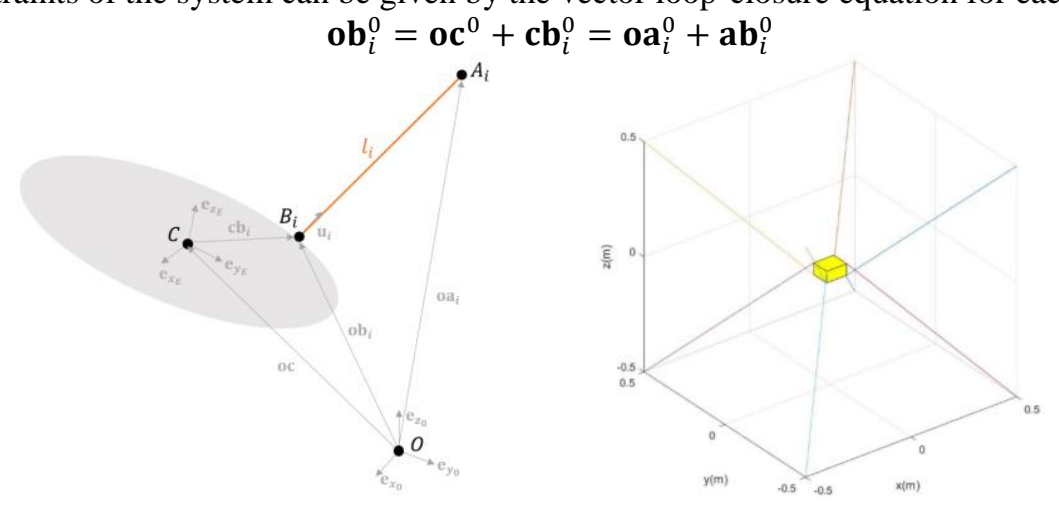

Fig. 1. Schematic representation of one cable (left) and robot with 8 cables view (right)

If the sagging effect is ignored, each cable is similar to a rigid rod when in static equilibrium and its length can be calculated by:

$$
l_{i}=\left\|\mathbf{a b}_{i}^{0}\right\|_{2}=\left\|-\mathbf{o} \mathbf{a}_{i}^{0}+\mathbf{o c}^{0}+\mathbf{R}_{O E} \mathbf{c b}_{i}^{E}\right\|_{2}
$$

with $\mathbf{R}_{O E}$ as the rotation matrix between the effector frame $\mathcal{R}_{E}$ and the fixed frame of reference $\mathcal{R}_{O}$. This equation gives the inverse kinematics of the robot.

Tab. 1. Anchor points position (mm)

\begin{tabular}{ccccccccc}
\hline $\boldsymbol{i}$ & $\mathbf{1}$ & $\mathbf{2}$ & $\mathbf{3}$ & $\mathbf{4}$ & $\mathbf{5}$ & $\mathbf{6}$ & $\mathbf{7}$ & $\mathbf{8}$ \\
\hline \multirow{2}{\mathbf{oa}_{\boldsymbol{i}}^{\mathbf{0}}}[\begin{array}{c}{500}\\
{500}\\
{-500}\end{array}]{} & {$\left[\begin{array}{l}500 \\
500 \\
500\end{array}\right]$} & {$\left[\begin{array}{c}-500 \\
500 \\
500\end{array}\right]$} & {$\left[\begin{array}{c}-500 \\
500 \\
-500\end{array}\right]$} & {$\left[\begin{array}{c}-500 \\
-500 \\
500\end{array}\right]$} & {$\left[\begin{array}{c}-500 \\
-500 \\
-500\end{array}\right]$} & {$\left[\begin{array}{c}500 \\
-500 \\
-500\end{array}\right]$} & {$\left[\begin{array}{c}500 \\
-500 \\
500\end{array}\right]$} \\
\hline $\mathbf{c b}_{\boldsymbol{i}}^{\boldsymbol{E}}$ & {$\left[\begin{array}{c}47.6 \\
40.2 \\
25.0\end{array}\right]$} & {$\left[\begin{array}{c}47.6 \\
40.2 \\
-25.0\end{array}\right]$} & {$\left[\begin{array}{c}-47.6 \\
40.2 \\
-25.0\end{array}\right]$} & {$\left[\begin{array}{c}-47.6 \\
40.2 \\
25.0\end{array}\right]$} & {$\left[\begin{array}{l}-47.6 \\
-40.2 \\
-25.0\end{array}\right]$} & {$\left[\begin{array}{c}-47.6 \\
-40.2 \\
25.0\end{array}\right]$} & {$\left[\begin{array}{c}47.6 \\
-40.2 \\
25.0\end{array}\right]$} & {$\left[\begin{array}{c}47.6 \\
-40.2 \\
-25.0\end{array}\right]$} \\
\hline
\end{tabular}

Table 1 indicates the anchor point parameters used in this study of a fully constrained 8-cable CDPR. This configuration is similar to that presented in [3].

At the preliminary design stage, it is crucial to have an estimation of the robot stiffness. The following paragraph outlines the computation of the stiffness matrix when cable preloads are considered.

\subsection{Stiffness matrix formulation with preload cables}

The 6x8 static Jacobian matrix, mapping the cable tensions to the wrench that cables apply on the mobile platform, has a rank of 6 and its kernel is 2-dimensional. Therefore, 
the static equilibrium of the robot can be achieved with infinite combinations of cable tensions. In order to formulate the stiffness matrix of the robot, potential energy is used to compute the stiffness matrix associated with one cable.

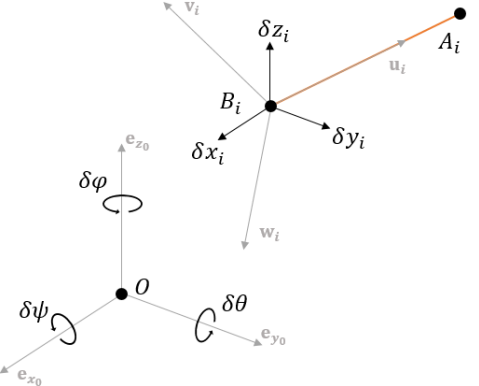

Fig. 2. Schematic representation of small variations

For a small variation of the configuration of the end-effector, there is a variation of the positions of points $B_{i}$ (figure 2):

$$
\Delta \mathbf{o b}_{i}^{0}=\Delta \mathbf{o c} \mathbf{c}^{0}+\Delta \boldsymbol{\theta}_{E}^{0} \times \mathbf{R}_{O E} \mathbf{c b}_{i}^{E}=\Delta \mathbf{o c} \mathbf{c}^{0}+\widetilde{\Delta \boldsymbol{\theta}_{E}^{0}} \mathbf{c b}_{i}^{0}=\Delta \mathbf{o c}{ }^{0}-\widetilde{\mathbf{c b}}_{l}^{0} \Delta \boldsymbol{\theta}_{E}^{0}
$$

With :

$$
\begin{gathered}
\Delta \mathbf{o b}_{i}^{0}=\left[\begin{array}{l}
\delta x_{i} \\
\delta y_{i} \\
\delta z_{i}
\end{array}\right] ; \quad \Delta \mathbf{o c}^{0}=\left[\begin{array}{c}
\delta x_{C} \\
\delta y_{C} \\
\delta z_{C}
\end{array}\right] ; \quad \Delta \boldsymbol{\theta}_{C}^{0}=\left[\begin{array}{c}
\delta \psi \\
\delta \theta \\
\delta \varphi
\end{array}\right] ; \quad \widetilde{\Delta \boldsymbol{\theta}_{C}^{0}}=\left[\begin{array}{ccc}
0 & -\delta \varphi & \delta \theta \\
\delta \varphi & 0 & -\delta \psi \\
-\delta \theta & \delta \psi & 0
\end{array}\right] ; \\
\mathbf{c b}_{i}^{0}=\left[\begin{array}{c}
X_{B i} \\
Y_{B i} \\
Z_{B i}
\end{array}\right] ; \widetilde{c}^{0}=\left[\begin{array}{ccc}
0 & Z_{B i} & -Y_{B i} \\
-Z_{B i} & 0 & X_{B i} \\
Y_{B i} & -X_{B i} & 0
\end{array}\right]
\end{gathered}
$$

We have :

$$
\left[\begin{array}{l}
\delta x_{i} \\
\delta y_{i} \\
\delta z_{i}
\end{array}\right]=\underbrace{\left[\begin{array}{lllllr}
1 & 0 & 0 & 0 & Z_{B i} & -Y_{B i} \\
0 & 1 & 0 & -Z_{B i} & 0 & X_{B i} \\
0 & 0 & 1 & Y_{B i} & -X_{B i} & 0
\end{array}\right]}_{\mathbf{H}_{i}}\left[\begin{array}{c}
\delta x_{C} \\
\delta y_{C} \\
\delta z_{C} \\
\delta \psi \\
\delta \theta \\
\delta \varphi
\end{array}\right] \leftrightarrow \Delta \mathbf{o b}_{i}^{0}=\mathbf{H}_{i}\left[\begin{array}{c}
\Delta \mathbf{o c}^{0} \\
\Delta \boldsymbol{\theta}_{C}^{0}
\end{array}\right]
$$

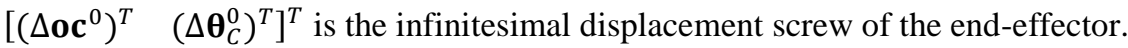

A displacement of $B_{i}$ from the cable with respect to its static equilibrium configuration in the reference $\mathcal{R}_{i}\left(A_{i}, \vec{u}_{i}, \vec{v}_{i}, \vec{w}_{i}\right)$ is given by $\Delta \mathbf{o b}_{i}^{i}=\left[\begin{array}{lll}\delta u_{i} & \delta v_{i} & \delta w_{i}\end{array}\right]^{T}$.

We have the relationship:

$$
\Delta \mathbf{o b}_{i}^{0}=\mathbf{R}_{O i} \Delta \mathbf{o b} \mathbf{b}_{i}^{i}
$$

where $\mathbf{R}_{O i}$ is the rotation matrix between the reference $\mathcal{R}_{i}$ and the fixed reference $\mathcal{R}_{O}$ :

$\mathbf{R}_{O i}=\left[\begin{array}{lll}\mathbf{u}_{i}^{0} & \mathbf{v}_{i}^{0} & \mathbf{w}_{i}^{0}\end{array}\right]$. We denote by $l_{0, i}$ the free length of the non-preloaded cable $i(i=1, \ldots, 8)$ and $l_{T i}$ its length stretched to the static equilibrium configuration. During small displacements, the current length of the cable is given by: 


$$
l_{i}=\sqrt{\left(L_{T i}+\delta u_{i}\right)^{2}+\delta v_{i}^{2}+\delta w_{i}^{2}}
$$

The deformation energy of a cable is then:

$$
V_{i}=\frac{1}{2} k_{i}\left(l_{i}-l_{0, i}\right)^{2}
$$

for a linear behavior of the cable: $k_{i}=\frac{E_{0} S}{l_{0, i}}$, with the elastic modulus $E_{0}$ and the cross section $S$. At the second order, the strain energy variation of the cable can be expressed as:

$$
V_{i}=\frac{1}{2}\left(\Delta \mathbf{o b}_{i}^{i}\right)^{T} \mathbf{K}_{i} \Delta \mathbf{o b}_{i}^{i}
$$

where $\mathbf{K}_{i}$ is the Hessian matrix at the equilibrium $\left(\Delta \mathbf{o} \mathbf{b}_{i}^{i}=\mathbf{0}\right)$ of $V_{i}$ with respect to the variables $\delta u_{i}, \delta v_{i}$ and $\delta w_{i}$.

$$
\mathbf{K}_{i}=\boldsymbol{\nabla} \boldsymbol{\nabla}^{T}\left(V_{i}\right)=\left[\begin{array}{ccc}
k_{i} & 0 & 0 \\
0 & \frac{T_{i}}{l_{T i}} & 0 \\
0 & 0 & \frac{T_{i}}{l_{T i}}
\end{array}\right]
$$

$\nabla$ is the gradient operator $\nabla^{T}=\left[\begin{array}{lll}\frac{\partial}{\partial\left(\delta u_{i}\right)} & \frac{\partial}{\partial\left(\delta v_{i}\right)} & \frac{\partial}{\partial\left(\delta w_{i}\right)}\end{array}\right]$ and $T_{i}$ is the cable preload.

The deformation energy of a cable can then be expressed as a function of the small displacements of the mobile platform:

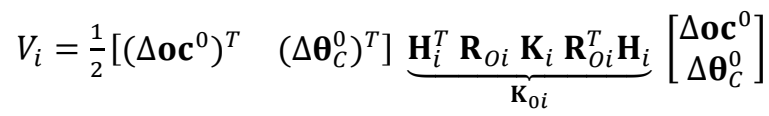

Hence the stiffness matrix of the cable robot:

$$
\mathbf{K}_{\text {robot }}=\sum_{i=1}^{m} \mathbf{K}_{0 i}
$$

The matrix $\mathbf{R}_{O i}^{T} \mathbf{H}_{i}$ can be detailed as follows:

$$
\mathbf{R}_{O i}^{T} \mathbf{H}_{i}=\left[\begin{array}{c}
\mathbf{u}_{i}^{0^{T}} \\
\mathbf{v}_{i}^{0^{T}} \\
\mathbf{w}_{i}^{0^{T}}
\end{array}\right]\left[\begin{array}{ll}
\mathbf{I}_{3} & -\widetilde{\mathbf{c}}_{l}^{0}
\end{array}\right]=\left[\begin{array}{cc}
\mathbf{u}_{i}^{0^{T}} & \mathbf{u}_{i}^{0^{T}} \cdot \widetilde{\mathbf{b}}_{l}^{0} \\
\mathbf{v}_{i}^{0^{T}} & \mathbf{v}_{i}^{0^{T}} \cdot \widetilde{\mathbf{b}}_{l}^{0} \\
\mathbf{w}_{i}^{0^{T}} & \mathbf{w}_{i}^{0^{T}} \cdot \mathbf{c b}_{l}^{0}
\end{array}\right]=\left[\begin{array}{cc}
\mathbf{u}_{i}^{0^{T}} & \left(\mathbf{c} \mathbf{b}_{i}^{0} \times \mathbf{u}_{i}^{0}\right)^{T} \\
\mathbf{v}_{i}^{0^{T}} & \left(\mathbf{c b} \mathbf{b}_{i}^{0} \times \mathbf{v}_{i}^{0}\right)^{T} \\
\mathbf{w}_{i}^{0^{T}} & \left(\mathbf{c b}_{i}^{0} \times \mathbf{w}_{i}^{0}\right)^{T}
\end{array}\right]
$$

With the Jacobian matrix:

$$
\begin{aligned}
& \mathbf{J}_{u}=\left[\begin{array}{ccc}
\mathbf{u}_{1}^{0} & \cdots & \mathbf{u}_{m}^{0} \\
\mathbf{c} \mathbf{b}_{1}^{0} \times \mathbf{u}_{1}^{0} & \cdots & \mathbf{c b _ { m } ^ { 0 }} \times \mathbf{u}_{m}^{0}
\end{array}\right], \mathbf{J}_{v}=\left[\begin{array}{ccc}
\mathbf{v}_{1}^{0} & \cdots & \mathbf{v}_{m}^{0} \\
\mathbf{c} \mathbf{b}_{1}^{0} \times \mathbf{v}_{1}^{0} & \cdots & \mathbf{c} \mathbf{b}_{m}^{0} \times \mathbf{v}_{m}^{0}
\end{array}\right], \mathbf{J}_{w}= \\
& {\left[\begin{array}{ccc}
\mathbf{w}_{1}^{0} & \cdots & \mathbf{w}_{m}^{0} \\
\mathbf{c b}_{1}^{0} \times \mathbf{w}_{1}^{0} & \cdots & \mathbf{c b}_{m}^{0} \times \mathbf{w}_{m}^{0}
\end{array}\right]}
\end{aligned}
$$


We can decompose the stiffness matrix with preloaded cables into a sum of three matrices:

$$
\mathbf{K}_{\text {robot }}=\mathbf{J}_{u}\left[\begin{array}{ccc}
k_{1} & 0 & 0 \\
0 & \ddots & 0 \\
0 & 0 & k_{8}
\end{array}\right] \mathbf{J}_{u}^{T}+\mathbf{J}_{v}\left[\begin{array}{ccc}
\frac{T_{1}}{l_{T 1}} & 0 & 0 \\
0 & \ddots & 0 \\
0 & 0 & T_{8} \\
l_{T 8}
\end{array}\right] \mathbf{T}_{v}^{T}+\mathbf{J}_{w}\left[\begin{array}{ccc}
\frac{T_{1}}{l_{T 1}} & 0 & 0 \\
0 & \ddots & 0 \\
0 & 0 & \frac{T_{8}}{l_{T 8}}
\end{array}\right] \mathbf{J}_{w}^{T}
$$

If the preload is zero, we obtained the same matrix express in [5].

\subsection{Mass matrix formulation and vibration modes}

Mass matrix formulation can be obtained from the kinetic energy of the mobile platform. The generalized coordinates of the system are denoted by $\mathbf{q}=(x, y, z, \psi, \theta, \varphi)$ with the angles defines by Tait-Bryan XYZ convention. The Kinetic energy is expressed by:

$$
T=\frac{1}{2} m_{e} \mathbf{v}_{C}^{0^{T}} \mathbf{v}_{C}^{0}+\frac{1}{2} \mathbf{\Omega}^{E^{T}} \cdot\left(\mathbf{I}_{C}^{E} \mathbf{\Omega}^{E}\right)
$$

where $\mathbf{v}_{C}^{0}$ is the translational velocity of the center of mass $C$ of the end-effector, $\boldsymbol{\Omega}^{E}$ is the end-effector rotational velocity expressed on the end-effector frame, $m_{e}$ and $\mathbf{I}_{C}^{E}$ are respectively the mass of the end-effector and its inertia matrix expressed in the frame $\mathcal{R}_{E}\left(C, \mathbf{e}_{x_{E}}, \mathbf{e}_{y_{E}}, \mathbf{e}_{z_{E}}\right)$. For infinitesimal displacements denoted by $\delta \mathbf{q}$ around a static equilibrium configuration, the kinetic energy can be expressed as follows:

where $\mathrm{M}$ is given by

$$
T=\frac{1}{2} \delta \dot{\mathbf{q}}^{T} \mathbf{M} \delta \dot{\mathbf{q}}
$$

$$
\mathbf{M}=\left[\begin{array}{cc}
m_{e} \mathbf{I}_{3} & \mathbf{0}_{3} \\
\mathbf{0}_{3} & \mathbf{R}_{O E} \mathbf{I}_{C}^{E} \mathbf{R}_{O E}^{T}
\end{array}\right]
$$

Having established the stiffness and the mass matrices $\mathbf{K}$ and $\mathbf{M}$, undamped vibration modes can be determined by the resolution of the following eigen problem:

$$
\left(\mathbf{K}-\omega_{0 i}^{2} \mathbf{M}\right) \mathbf{p}_{i}=\mathbf{0}
$$

where $\omega_{0 i}(i=1, \ldots, 6)$. are the natural pulsations associated with the modal vectors (mode shapes) $\mathbf{p}_{i}$.

\section{$2.4 \quad$ Non-linear dynamic modelling}

In order to study the behavior of the robot in large displacements (large rotations) and subject to dynamic loading due to inertia and cable forces, non-linear effects must be considered such as gyroscopic coupling and cable sagging. The actual performance of the robot in terms of precision and vibration stability in operating conditions cannot be predicted precisely by the linear elasto-dynamic model developed in the previous section, where infinitesimal displacement assumption have been adopted. A non-linear dynamic model is therefore required to simulate the robot's movements and estimate its performance during path tacking.

For a totally constrained cable robot with all the cables working under tension, we can assume that the cable works like a traction spring with a negligible mass. The tension 
force generated by the cable is expressed in equation 16 as a function of the following cable parameters: the elastic modulus $E_{0}$, the cross section $S$, the free length $l_{0, i}$ the current length $l_{i}$.

$$
T_{\text {elas }, i}=E_{0} S\left(\frac{l_{i}}{l_{0, i}}-1\right)
$$

The relation between the preloaded tension $T_{i}$ and its corresponding cable elongation $l_{T i}$ is given by the equation:

$$
T_{i}=E_{0} S\left(\frac{l_{T i}}{l_{0, i}}-1\right)
$$

By replacing the cable free length $l_{0, i}$ from the equation (17) into equation (16), the tension force becomes :

$$
T_{\text {elas }, i}=\frac{l_{i}}{l_{T i}}\left(T_{i}+E_{0} S\right)-E_{0} S
$$

However, cables work only under tension. Hence, the force in a cable is null or positive, which means:

$$
\left\{\begin{array}{c}
F_{i}=T_{\text {elas }, i} \text { if } T_{\text {elas }, i} \geq 0 \\
F_{i}=0 \text { if } T_{\text {elas }, i}<0
\end{array}\right.
$$

The dynamics of the robot can be formulated with Lagrange equations. We use the expression of the kinetic energy given by equation (14). The generalized force, relative to each generalized coordinate, is given by:

$$
P_{q_{j}}=F_{q_{j}}+\sum_{i=1}^{m} F_{i} \mathbf{u}_{i}^{0^{T}} \cdot \frac{\partial}{\partial q_{j}}\left(\mathbf{o b}_{i}^{0}\right)
$$

Where $F_{i}$ is the force created by the cable tension, $\mathbf{u}_{i}^{0}$ and the partial derivative of the vector $\mathbf{o b}_{i}^{0}$ dependent on the generalized coordinate $\frac{\partial}{\partial q}\left(\mathbf{o b}_{i}^{0}\right) . F_{q j}(j=1, \ldots, 8)$ denotes the other external forces projected on the generalized coordinate such as the weight of the end-effector.

The Dynamic equations are obtained as follows:

$$
\mathcal{L}_{q_{j}}: \frac{d}{d t} \frac{\partial T}{\partial \dot{q}_{j}}-\frac{\partial T}{\partial q_{j}}=P_{q_{j}}
$$

This differential equation is solved with ode45 from Matlab software by using $\mathbf{Y}=$ $\left[\begin{array}{ll}\mathbf{q}^{T} & \dot{\mathbf{q}}^{T}\end{array}\right]^{T}$ as a state vector and by controlling the cable preload length $l_{T i} . l_{i}$ is calculated with the direct kinematic model.

\section{Simulation results}

The simulations are performed with cable diameter of $1.5 \mathrm{~mm}$ and for two cases of cable material: nylon cables with an elastic modulus of $3.9 \times 10^{9} \mathrm{~N} . \mathrm{m}^{-2}$ and steel cables with an elastic modulus of $102 \times 10^{9} \mathrm{~N} . \mathrm{m}^{-2}$. The mobile platform is assimilated to a homogenous parallelepiped of size $(95.1 \mathrm{~mm}, 80.5 \mathrm{~mm}, 50.0 \mathrm{~mm})$ with a mass of $1 \mathrm{~kg}$. In these simulations, the orientation of the mobile platform is maintained constant at $\left(0^{\circ}, 0^{\circ}, 0^{\circ}\right)$. 


\subsection{Static and modal analysis}

The static analysis is performed over all the feasible workspace. The stiffness matrix of the robot is that which relates the infinitesimal displacements twist of the mobile platform to the external force wrench applied to it at its characteristic point $C$. In our case, the cables are assumed to be permanently preloaded so as to avoid a discontinuity of the solution. If the cable becomes slack, the analysis is no longer valid.

The mapping of the rigidity terms is shown in Fig. 3 for the layers $z=0 m$ and $z=$ $0.25 \mathrm{~m}$ for the steel cable case. It can be seen that the stiffness depends on effector position. The translational rigidities in $X$ and $Y$ directions, $k_{x x}$ and $k_{y y}$, are between $400 \mathrm{kN} / \mathrm{m}$ and $550 \mathrm{kN} / \mathrm{m}$. The translational rigidity in $Z$ direction, $k_{z Z}$, is higher and varies between $700 \mathrm{kN} / \mathrm{m}$ and $900 \mathrm{kN} / \mathrm{m}$. The rotational rigidities around $X, Y$ and $Z$ directions, $k_{\psi \psi}, k_{\theta \theta}$ and $k_{\varphi \varphi}$, vary respectively between $2.1 \mathrm{kN} / \mathrm{rad}$ and $2.8 \mathrm{kN} / \mathrm{rad}$, $2.7 \mathrm{kN} / \mathrm{rad}$ and $5 \mathrm{kN} / \mathrm{rad}$, and $0.1 \mathrm{kN} / \mathrm{rad}$ and $0.9 \mathrm{kN} / \mathrm{rad}$. The rotational rigidity $k_{\varphi \varphi}$ is the lowest because a small rotation around the $\mathrm{Z}$ axis produces displacements of the anchor points almost orthogonal to the cables. In this study, only the diagonal terms of the rigidity matrix are analyzed.
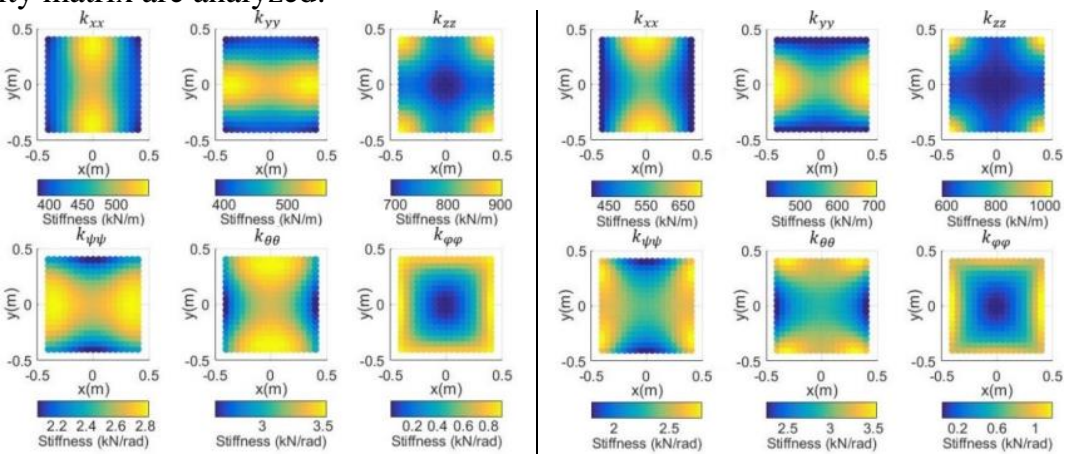

Fig. 3. Stiffness on the plane $x y$ for $z=0 m$ (left) and for $z=0.25 m$ (right) for a steel cable
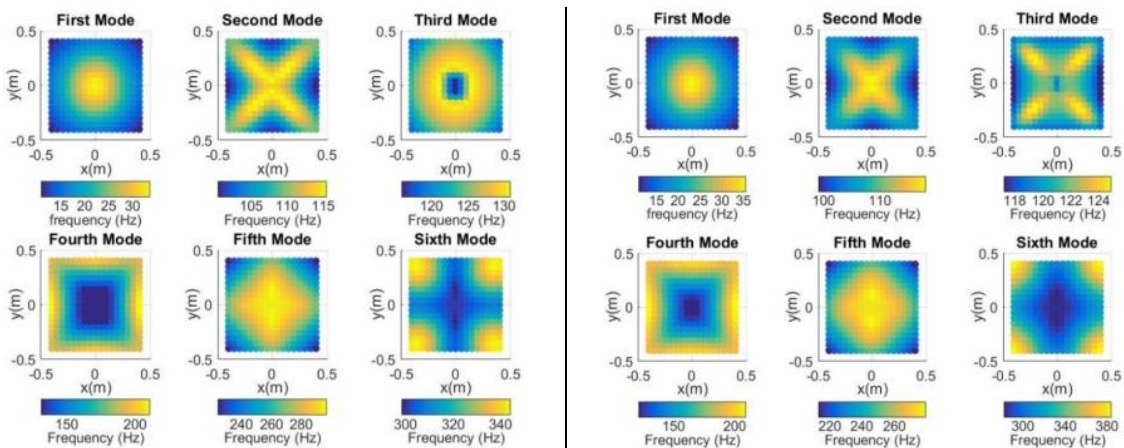

Fig. 4. Natural mode on the plane $x y$ for $z=0 m$ (left) and for $z=0.25 m$ (right) for a steel cable

The vibration cartography is shown in Fig 4 for the layer $z=0 \mathrm{~m}$ and $z=0.25 \mathrm{~m}$ for a steel cable. The minimum first mode is closed to $10 \mathrm{~Hz}$ when the effector is at the workspace extremity. In the center of the workspace, the first natural frequency is above $30 \mathrm{~Hz}$. 
Tab. 2. Influence of the preload on the stiffness of the position $(0,0,0)$ for a nylon cable

\begin{tabular}{cllllll}
\hline$T_{i}$ & $k_{x x}(\mathrm{kN} / \mathrm{m})$ & $k_{y y}(\mathrm{kN} / \mathrm{m})$ & $k_{z z}(\mathrm{kN} / \mathrm{m})$ & $k_{\psi \psi}\left(\mathrm{N} /{ }^{\circ}\right)$ & $k_{\theta \theta}\left(\mathrm{N} /{ }^{\circ}\right)$ & $k_{\varphi \varphi}\left(\mathrm{N} /{ }^{\circ}\right)$ \\
\hline $0 \mathrm{~N}$ & 19.618 & 20.259 & 26.415 & 1.7790 & 2.2012 & 0.0225 \\
\hline \multirow{2}{*}{$50 \mathrm{~N}$} & 19.954 & 20.590 & 26.702 & 1.7849 & 2.2094 & 0.0546 \\
& $1.68 \%$ & $1.61 \%$ & $1.08 \%$ & $0.33 \%$ & $0.37 \%$ & $58.89 \%$ \\
\hline \multirow{2}{*}{$100 \mathrm{~N}$} & 20.286 & 20.917 & 26.985 & 1.7907 & 2.2174 & 0.0863 \\
& $3.29 \%$ & $3.15 \%$ & $2.11 \%$ & $0.65 \%$ & $0.73 \%$ & $73.99 \%$ \\
\hline \multirow{2}{*}{$200 \mathrm{~N}$} & 20.935 & 21.557 & 27.539 & 1.8020 & 2.2333 & 0.1484 \\
& $6.29 \%$ & $6.02 \%$ & $4.08 \%$ & $1.28 \%$ & $1.44 \%$ & $84.87 \%$ \\
\hline
\end{tabular}

Tab. 3. Influence of the preload on the stiffness of the position $(0,0,0)$ for a steel cable

\begin{tabular}{cllllll}
\hline$T_{i}$ & $k_{x x}(\mathrm{kN} / \mathrm{m})$ & $k_{y y}(\mathrm{kN} / \mathrm{m})$ & $k_{z z}(\mathrm{kN} / \mathrm{m})$ & $k_{\psi \psi}\left(\mathrm{N} /{ }^{\circ}\right)$ & $k_{\theta \theta}\left(\mathrm{N} /{ }^{\circ}\right)$ & $k_{\varphi \varphi}\left(\mathrm{N} /{ }^{\circ}\right)$ \\
\hline $0 \mathrm{~N}$ & 513.09 & 529,85 & 690.84 & 46.5278 & 57.5688 & 0.5872 \\
\hline \multirow{2}{*}{$50 \mathrm{~N}$} & 513.43 & 530,18 & 691.13 & 46.5337 & 57.5771 & 0.6196 \\
& $0.07 \%$ & $0.06 \%$ & $0.04 \%$ & $0.01 \%$ & $0.01 \%$ & $5.23 \%$ \\
\hline \multirow{2}{*}{$100 \mathrm{~N}$} & 513.77 & 530,52 & 691.42 & 46.5396 & 57.5853 & 0.6520 \\
& $0.13 \%$ & $0.13 \%$ & $0.08 \%$ & $0.03 \%$ & $0.03 \%$ & $9.93 \%$ \\
\hline \multirow{2}{*}{$200 \mathrm{~N}$} & 514,45 & 531,18 & 692.00 & 46.5515 & 57.6018 & 0.7166 \\
& $0.26 \%$ & $0.25 \%$ & $0.17 \%$ & $0.05 \%$ & $0.06 \%$ & $18.06 \%$ \\
\hline
\end{tabular}

The influence of the preload on the robot stiffness is shown in Table 2 for a nylon cable and Table 3 for a steel cable at the position $(0 \mathrm{~m}, 0 \mathrm{~m}, 0 \mathrm{~m})$. For the same cross section, the steel stiffness is higher than the nylon stiffness. On the $\mathrm{x}$ axis, the stiffness is $513.09 \mathrm{kN} / \mathrm{m}$ for steel and $19.618 \mathrm{kN} / \mathrm{m}$ for nylon without preload. On the $\mathrm{x}$ axis, the stiffness increases by $6.29 \%$ for the nylon with a $200 \mathrm{~N}$ preload. For the steel cable, the preload has less influence with an increase of $0.26 \%$ on the $\mathrm{x}$ axis stiffness with a $200 \mathrm{~N}$ preload. This may be explained by the fact that a higher preload is required for steel cable to reach the same relative level of lateral stiffness as in nylon cable.The preload has a more significant impact on the lesser stiffness direction which is the $\varphi$ orientation (rotation on $\mathrm{z}$ ). For the nylon, the stiffness is increased by about $84.87 \%$ with a preload of $200 \mathrm{~N}$. For the steel, the stiffness is increased by about $18.06 \%$ with a preload of $200 \mathrm{~N}$.

Tab. 4. Influence of the preload on the natural frequency of the position $(0,0,0)$ for a nylon cable

\begin{tabular}{cllllll}
\hline \multicolumn{1}{c}{$T_{i}$} & \multicolumn{1}{c}{$f_{1}(\mathrm{~Hz})$} & $f_{2}(\mathrm{~Hz})$ & $f_{3}(\mathrm{~Hz})$ & \multicolumn{1}{c}{$f_{4}(\mathrm{~Hz})$} & \multicolumn{1}{c}{$f_{5}(\mathrm{~Hz})$} & \multicolumn{1}{c}{$f_{6}(\mathrm{~Hz})$} \\
\hline $0 \mathrm{~N}$ & 6.6015 & 22.2921 & 22.6531 & 25.8668 & 57.6235 & 58.7635 \\
\hline \multirow{2}{*}{$50 \mathrm{~N}$} & 10.2964 & 22.4823 & 22.8377 & 26.0070 & 57.7307 & 58.8605 \\
& $35.89 \%$ & $0.85 \%$ & $0.81 \%$ & $0.54 \%$ & $0.19 \%$ & $0.16 \%$ \\
\hline \multirow{2}{*}{$100 \mathrm{~N}$} & 12.9446 & 22.6682 & 23.0183 & 26.1445 & 57.8362 & 58.9560 \\
& $49 \%$ & $1.66 \%$ & $1.59 \%$ & $1.06 \%$ & $0.37 \%$ & $0.33 \%$ \\
\hline \multirow{2}{*}{$200 \mathrm{~N}$} & 16.9720 & 23.0278 & 23.3677 & 26.4117 & 58.0421 & 59.1425 \\
& $61.1 \%$ & $3.19 \%$ & $3.06 \%$ & $2.06 \%$ & $0.72 \%$ & $0.64 \%$ \\
\hline
\end{tabular}

The influence of the preload on the natural frequency is shown in the tables 4 and 5 . The first natural frequency is lower for the nylon cable with $6.6015 \mathrm{~Hz}$ than the steel cable with $33.7605 \mathrm{~Hz}$. The preload effect on the frequency is more significant on nylon cable by an increase of the first mode by $61.1 \%$ when preloaded at $200 \mathrm{~N}$ preload. The preload has a significant impact on the first mode. For steel cable, the first mode is 
increased by $9.48 \%$ with a $200 \mathrm{~N}$ preload, but the other modes are increased by less than $0.13 \%$.

Tab. 5. Influence of the preload on the natural frequency of the position $(0,0,0)$ for a steel cable

\begin{tabular}{clllrrl}
\hline \multicolumn{1}{c}{$T_{i}$} & $f_{1}(\mathrm{~Hz})$ & $f_{2}(\mathrm{~Hz})$ & $f_{3}(\mathrm{~Hz})$ & $f_{4}(\mathrm{~Hz})$ & $f_{5}(\mathrm{~Hz})$ & \multicolumn{1}{c}{$f_{6}(\mathrm{~Hz})$} \\
\hline $0 \mathrm{~N}$ & 33.7605 & 114.0036 & 115.8499 & 132.2848 & 294.6912 & 300.5215 \\
\hline \multirow{2}{*}{$50 \mathrm{~N}$} & 34.6791 & 114.0412 & 115.8864 & 132.3125 & 294.7123 & 300.5406 \\
& $2.65 \%$ & $0.033 \%$ & $0.0315 \%$ & $0.0209 \%$ & $0.0072 \%$ & $0.0064 \%$ \\
\hline \multirow{2}{*}{$100 \mathrm{~N}$} & 35.5736 & 114.0788 & 115.9229 & 132.3402 & 294.7335 & 300.5597 \\
& $5.1 \%$ & $0.0659 \%$ & $0.0629 \%$ & $0.0418 \%$ & $0.0143 \%$ & $0.0127 \%$ \\
\hline \multirow{2}{*}{$200 \mathrm{~N}$} & 37.2969 & 114.1538 & 115.9957 & 132.3955 & 294.7756 & 300.5979 \\
& $9.48 \%$ & $0.13 \%$ & $0.13 \%$ & $0.0836 \%$ & $0.0286 \%$ & $0.0254 \%$ \\
\hline
\end{tabular}

\subsection{Dynamic simulation for a given position}

A first dynamic analysis is done on a given position at $(0 \mathrm{~m}, 0 \mathrm{~m}, 0 \mathrm{~m})$. An initial perturbation is done by an initial translational velocity of $(0.5 \mathrm{~m} / \mathrm{s}, 0.5 \mathrm{~m} / \mathrm{s}, 0.5 \mathrm{~m} / \mathrm{s})$ and rotational velocity of $(0.1 \mathrm{rad} / \mathrm{s}, 0.1 \mathrm{rad} / \mathrm{s}, 0.1 \mathrm{rad} / \mathrm{s})$. Two simulations are carried out in order to study the induced vibrations. It is not possible to analyze the Fourier transform of cable working only under traction stress without preload, which may be explained by the elastic force discontinuity. Cable preload is added with a force distribution algorithm. The close-form algorithm[11] is used to compute the force distribution with $t_{\text {min }}=200 \mathrm{~N}$ and $t_{\max }=300 \mathrm{~N}$. With the preload, the static natural frequencies from table 5 are found. The first mode is presented on the $\varphi$ rotation, which is the lesser stiffness. The second and the third modes are closed and they are presented respectively on the $\mathrm{x}$ and $\mathrm{y}$ translations. The fourth mode is on the $\mathrm{z}$ translation. The fifth and sixth modes are respectively on the $\psi$ and $\theta$ rotations. These results show that, at the position $(0 \mathrm{~m}, 0 \mathrm{~m}, 0 \mathrm{~m})$, the two highest modal frequencies correspond to rotations of the mobile platform around the $\mathrm{x}$ and $\mathrm{y}$ axes respectively. At this position, movements are clearly decoupled. However, for other positions, the movements are coupled. The first mode presented mainly a rotation around the $\mathrm{z}$ axis coupled with a translation in the xy plane.

\subsection{Dynamic simulation for a circular trajectory}

In order to simulate the behavior during the movement of the effector, a circular trajectory is considered. The angular parameter of the trajectory is calculated with a polynomial function:

$$
\alpha(t)=\left[\begin{array}{llll}
t^{3} & t^{2} & t & 1
\end{array}\right]\left[\begin{array}{cccc}
2 / T^{3} & 1 / T^{2} & -2 / T^{3} & 1 / T^{2} \\
-3 / T^{2} & -2 / T & 3 / T^{2} & -1 / T \\
0 & 1 & 0 & 0 \\
1 & 0 & 0 & 0
\end{array}\right]\left[\begin{array}{c}
\alpha_{\text {init }} \\
\dot{\alpha}_{\text {init }} \\
\alpha_{\text {end }} \\
\dot{\alpha}_{\text {end }}
\end{array}\right]
$$

where $T$ is the period of the circular trajectory, $\alpha_{\text {init }}$ is the initial angle position, $\dot{\alpha}_{\text {init }}$ is the initial angle velocity, $\alpha_{\text {end }}$ is the final angle position and $\dot{\alpha}_{\text {end }}$ the final velocity. The circular trajectory follows the equations:

$$
x=r \cos (\alpha), y=r \sin (\alpha), z=0
$$


The polynomial function $\alpha(t)$ is used to be sure that the initial acceleration is null with an initial velocity $\dot{\alpha}_{\text {init }}$ also null. The simulated trajectory is a circle of radius $33 \mathrm{~mm}$ with a 1 -second period that represents a path velocity of $207 \mathrm{~mm} / \mathrm{s}$. The movement of the platform is controlled by the variation of cable lengths calculated using the inverse geometric model. Disturbances are caused solely by the elastodynamic behavior of the robot.

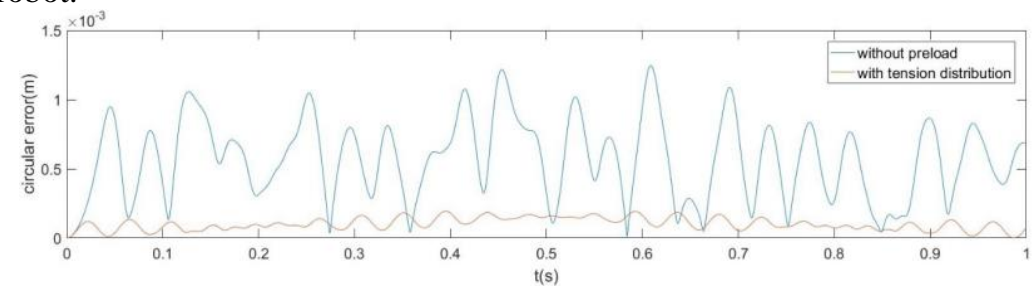

Fig. 5. Circular error with nylon cable for a circular trajectory

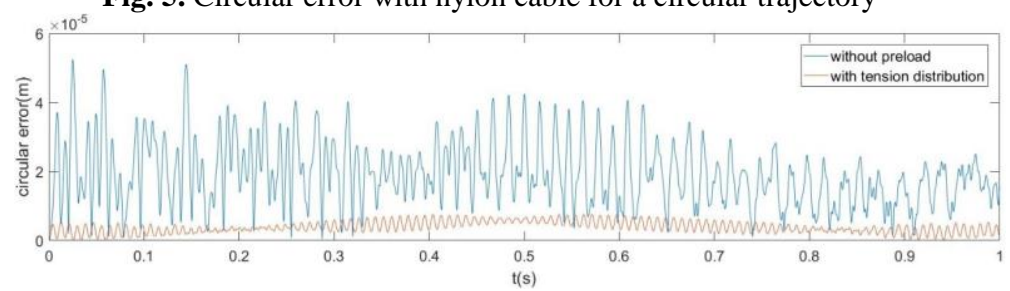

Fig. 6. Circular error with steel cable for a circular trajectory

The position error is shown on figure 5 for a nylon cable and figure 6 for a steel cable. Simulation without preload and with a preload determined with a force distribution algorithm are shown. The close-form algorithm[11] was used to compute the force distribution with $t_{\text {min }}=10 \mathrm{~N}$ and $t_{\max }=20 \mathrm{~N}$. The position error is higher for the nylon cable than steel. The maximum circular error is $52.5 \mu \mathrm{m}$ for steel cable and $1.2 \mathrm{~mm}$ for nylon without preload. The error is lower when using the force distribution algorithm. The maximum circular error is $7.6 \mu \mathrm{m}$ for steel cable and $194 \mu \mathrm{m}$ for nylon with preloads. The dynamic performance is better with a preload on the cable.

\section{Conclusion}

The influence of the preload of the cables on the static and dynamic behavior has been studied. This has been done by computing the robot stiffness matrix from a second order approximation of the deformation energy of preloaded cables, expressed as a function of infinitesimal displacement coordinates.

In terms of static and modal behavior, the preload has a significant impact mainly on the rotational stiffness around the $\mathrm{Z}$ axis and the first natural frequency. For a dynamic trajectory, the preload plays a significant role. It keeps the cables under tension and prevents the discontinuity due to slack cables. Moreover, the preload improves the trajectory accuracy.

The preload influence is more significant with a low elastic module. For instance, the preload impact is more significant with the nylon cable than the steel cable. This result may seem intuitive, but it remains necessary to quantify the gain in terms of rigidity and natural frequencies in order to optimize the choice of cables. In the literature, rigid cables are generally chosen. 
The developed dynamic model can be used to choose the preload on the cable as well as to determine the minimal preload to avoid slack cable. This study has been carried out for a constant orientation of the mobile platform, but the developments presented herein should be exploited to investigate the workspace at different orientations. However, the dynamic model chosen considers only the cable elasticity. Future works need to integrate the viscoelastic behavior of the cables. The damping effect can help to stabilize and reduce the oscillations amplitude, especially for low stiffness cables. Although this study was undertaken on a totally constrained cable robot, it may be extended to other CDPR applications.

\section{Acknowledgements}

This paper is part of the CABFAB project funded by the Auvergne-Rhône-Alpes Region as part of the 2017 Ambition Pack Research program.

\section{References}

1. Gosselin, C.: Cable-driven parallel mechanisms: state of the art and perspectives. Mech. Eng. Rev. 1, DSM0004-DSM0004 (2014).

2. Gagliardini, L., Caro, S., Gouttefarde, M., Wenger, P., Girin, A.: A Reconfigurable Cable-Driven Parallel Robot for Sandblasting and Painting of Large Structures. In: Pott, A. and Bruckmann, T. (eds.) Cable-Driven Parallel Robots. pp. 275-291. Springer International Publishing, Cham (2015).

3. Pott, A., Mütherich, H., Kraus, W., Schmidt, V., Miermeister, P., Verl, A.: IPAnema: A family of Cable-Driven Parallel Robots for Industrial Applications. In: Bruckmann, T. and Pott, A. (eds.) Cable-Driven Parallel Robots. pp. 119-134. Springer Berlin Heidelberg, Berlin, Heidelberg (2013).

4. Landsberger, S., Sheridan, T.: Parallel Link Manipulators, (1987).

5. Diao, X., Ma, O.: Vibration analysis of cable-driven parallel manipulators. Multibody Syst. Dyn. 21, 347-360 (2009).

6. Tourajizadeh, H.: Longitudinal and Lateral Vibration Analysis of Cables in a Cable Robot using Finite Element Method. 10, (2017).

7. Yuan, H., Courteille, E., Deblaise, D.: Static and dynamic stiffness analyses of cabledriven parallel robots with non-negligible cable mass and elasticity. Mech. Mach. Theory. 85, 64-81 (2015).

8. Yuan, H., Courteille, E., Gouttefarde, M., Hervé, P.E.: Vibration analysis of cabledriven parallel robots based on the dynamic stiffness matrix method. J. Sound Vib. 394, 527-544 (2017).

9. Weber, X., Cuvillon, L., Gangloff, J.: Active vibration canceling of a cable-driven parallel robot using reaction wheels. 2014 IEEE/RSJ Int. Conf. Intell. Robot. Syst. 1724-1729 (2014).

10. Rushton, M., Khajepour, A.: Transverse vibration control in planar cable-driven robotic manipulators. Mech. Mach. Sci. 53, 243-253 (2018).

11. Pott, A., Bruckmann, T., Mikelsons, L.: Closed-form Force Distribution for Parallel Wire Robots. In: Computational Kinematics. pp. 25-34 (2009). 